Gefässchirurgie 2009 · 14:24-30 DOI 10.1007/s00772-008-0650-4

Online publiziert: 23. Januar 2009

(c) Springer Medizin Verlag 2009

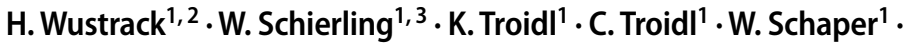 \\ T. Schmitz-Rixen ${ }^{1,2}$ \\ ${ }^{1}$ Arteriogenese-Forschungsgruppe des Max-Planck-Instituts \\ für Herz- und Lungenforschung, Bad Nauheim \\ ${ }^{2}$ Klinik für Gefäß- und Endovaskular-Chirurgie, Goethe-Universität Frankfurt am Main \\ ${ }^{3}$ Abteilung für Gefäß- und Endovaskuläre Chirurgie, Universität Regensburg
}

\section{Stimulierung des Wachstums peripherer und zerebraler Kolla- teralarterien zur Erhöhung der intravasalen Flussrate im Tiermodell}

Die Entwicklung von Kollateralen bei Patienten mit vaskulären Verschlusskrankheiten wird als Arteriogenese bezeichnet. Jedoch ist das Kollateralarterienwachstum in vielen Fällen nicht ausreichend, um die bestehenden Stenosen oder Verschlüsse vollständig zu kompensieren. Aus Tiermodellen wissen wir, dass durch das natürliche Wachstum von Kollateralarterien gerade einmal 40\% der maximalen Konduktanz der ursprünglichen Arterie erreicht werden $[1,3]$. Aus diesem Grund ist es ein wichtiges therapeutisches Ziel, nach Möglichkeiten zu suchen, das Wachstum von Kollateralarterien zu stimulieren und damit diesen natürlichen Prozess zu optimieren. Die klinische Anwendung von bekannten Wachstumsfaktoren wie „monocyte chemoattractant protein-1" (MCP-1) [6], „Vascular endothelial growth factor" (VEGF) $[1,2,5]$, „basic fibroblast growth factor" (bFGF), „fibroblast growth factor 2" oder 4 (FGF-2, FGF-4) werden jedoch kontrovers diskutiert [10, 13].

Auch unsere eigenen experimentellen Daten zeigten, dass eine lokale, intrakollaterale Anwendung von bFGF, MCP-1 oder FGF-4 nur zu einer teilweisen Kompensation der Konduktanz von okkludierten Hauptarterien durch das sich bildende Kollateralgefäßsystem führt. Dies deutet darauf hin, dass trotz großer Fortschritte im Verständnis der molekularen Mechanismen der Arteriogenese die eigentlichen Schlüssel- oder Mastergene, welche das
Kollateralwachstum auslösen, noch weitestgehend unbekannt sind.

In früheren Studien an einem Schweineund Kaninchenmodell konnte unsere Arbeitsgruppe zeigen, dass nach Femoralarterienligatur die Schubspannung die treibende Kraft der peripheren Arteriogenese ist und dass durch eine langfristige Erhöhung der Schubspannung durch eine Ableitung des Kollateralflusses in das Venensystem im Sinne einer arteriovenösen Fistel eine derartige Steigerung des Kollateralarterienwachstums zu erreichen ist, dass die physiologische Funktion der verschlossenen Arterie komplett wiederhergestellt werden kann $[1,3]$. Da in dieser Situation die molekularen Vorgänge maximal stimuliert sind, eignete sich das Modell hervorragend zur genomweiten Expressionsanalyse wachsender Kollateralarterien. Hierbei wurden u. a. zwei Gene identifiziert und charakterisiert, die an der Signalübertragung des physikalischen Stimulus in eine zelluläre Antwort beteiligt sind und deren gezielte Aktivierung oder Überexpression die Schubspannung simulieren bzw. ersetzen kann.

Es handelt sich zum einen um den mechanosensitiven Kalziumkanal transient receptor potential cation channel, subfamily $V$, member 4 (TRPV4), der bisher für die Regulation des Vasotonus verantwortlich gemacht wurde. Wir konnten seine Rolle beim aktiven Wachstumsprozess von Kollateralarterien nachweisen [11]. Das ande- re Gen kodiert für das actin-binding Rho activating protein (ABRA) und spielt möglicherweise eine Rolle beim notwendigen Umbau der Arteriolen [3].

Die funktionale Bedeutung von TRPV 4 für die Arteriogenese konnten wir mittels Modulation seiner Aktivität zeigen. Ein lokaler, intrakollateraler Gentransfer von ABRA im einfachen Ligaturmodell am Hinterlauf von Kaninchen steigerte ebenfalls die maximale kollaterale Konduktanz nach 7 Tagen um $67,3 \%( \pm 10,3 \%)$.

\section{Ein neuer Signalweg der Arteriogenese}

Aus diesen Arbeiten konnte ein neuer Signalweg der Arteriogenese formuliert werden: Der Verschluss einer Hauptarterie führt zu erhöhter Schubspannung im Kollateralkreislauf. Diese aktiviert den mechanosensitiven $\mathrm{Ca}^{2+}$-Kanal TRPV 4 durch den ein $\mathrm{Ca}^{2+}$-Einstrom in die Zelle erfolgt. Die Analyse des ABRA Promotors ergab Hinweise auf die $\mathrm{Ca}^{2+}$-abhängige Regulation seiner Genexpression. Er enthält neben Bindestellen für die Transkriptionsfaktoren Mef2c und Atf 4 auch eine Bindestelle (DRE-Site) für den transkriptionalen Repressor DREAM. Eine erhöhte $\mathrm{Ca}^{2+}$-Konzentration im Nukleus

Die Autorinnen H. Wustrack und W. Schierling haben gleichermaßen und zu gleichen Teilen an der Publikation mitgewirkt. 
löst diesen Repressor und eine Transkription kann stattfinden und das Kollateralwachstum ist initiiert (nicht publizierte Daten).

\section{Zielsetzung}

Ziel der weiteren Forschung ist nun mit der detaillierten Aufschlüsselung des Signalwegs der schubspannungsstimulierten Arteriogenese alternative Ansatzpunkte für die Therapie von Blutgefäßverschlüssen zu finden. Anhand der Arbeitshypothese sollen die molekularen Vorgänge stromab- und stromaufwärts der identifizierten Gene TRPV4 und ABRA untersucht werden. Von der Modulation dieser beiden Faktoren wird ein entscheidender Einfluss auf die Arteriogenese erwartet.

\section{Zerebrale Kollateralen}

Das Wachstum von Kollateralarterien spielt dabei nicht nur bei der Überbrückung von peripheren Stenosen und Verschlüssen eine wichtige Rolle. In klinischen Studien wurde auch die Bedeutung einer adäquaten hämodynamischen Kompensation durch den Circulus arteriosus Willisii bei Patienten mit hochgradiger Stenose oder Verschluss der A. carotis interna herausgestellt.

Der nächste folgerichtige Schritt war es dann, an der Ratte ein experimentelles Modell zur Erhöhung der intravasalen Flussrate und damit der Schubspannung im Bereich der zerebralen Kollateralzirkulation zu etablieren und zu analysieren, ob auf diese Weise das Wachstum zerebraler Kollateralarterien gesteigert werden kann. Die Entwicklung geschah aus zwei Gründen: Uns interessierte erstens, ob wir die gleichen Wachstumsmechanismen in einem anderen Kollateralgebiet finden konnten, deren Arterien zudem phylogenetisch aus einem anderen Keimblatt entstammen. Außerdem wollten wir durch dieses Modell die zuweilen kritisierte Kontamination der Kollateralgefäßpräparate mit quergestreifter Muskulatur vermeiden.

Die Bedeutung der in „micro arrays“ gefundenen Gensequenzen bedürfen der weiteren Analyse in gendefizienten Tiermodellen. Dies ist zur Zeit nur in der Maus realisierbar. Damit wurde es not-

Gefässchirurgie 2009 · 14:24-30 DOI 10.1007/s00772-008-0650-4

(c) Springer Medizin Verlag 2009

\section{H. Wustrack · W. Schierling · K. Troidl · C. Troidl · W. Schaper · T. Schmitz-Rixen Stimulierung des Wachstums peripherer und zerebraler Kollateralarterien zur Erhöhung der intravasalen Flussrate im Tiermodell}

\section{Zusammenfassung}

Hintergrund. Die Entwicklung einer Kollateralzirkulation (Arteriogenese) als Bypass des Verschlusses einer Leitarterie ist selbstlimitierend. In Modellen eines chronisch erhöhten Blutflusses hat das Expressionsprofil wachsender Kollateralen unsere Kenntnisse über den molekularen Mechanismus dieses Gefäßwachstums erweitert.

Material und Methoden. Die funktionelle Analyse erfordert den Einsatz gendefizienter Mausmodelle. Unsere entwickelten Modelle der Schubspannungserhöhung in der peripheren und Zerebralzirkulation werden mittels Cuff-Anastomosentechnik auf das Mausmodell übertragen. Nach zentraler Ligatur der A. femoralis superficialis bzw. der A. carotis communis wird der periphere Kollateralfluss in die V. femoralis bzw. V. jugularis gefistelt. Zur Erhöhung des zerebralen Blutflusses entwickeln wir analog zu einem Rattenmodell die einseitige Anlage einer AV-Fistel (Solo-Shunt-Modell) und die beidseitige Karotisligatur mit nachfolgender einseitiger AV-Fistel-Anlage (Ligatur-Shunt-Modell).

Ergebnisse. Die chronische Steigerung der Flussraten und Schubspannung vom Schweinemodell über ein Kaninchen- und Rattenmodell bis hin zu Mausmodellen ist mit hoher Erfolgsrate gelungen.

Schlussfolgerung. Die neue Anastomosentechnik ermöglicht den Einsatz gendefizienter Tiermodelle zur Aufklärung der molekularen Mechanismen der Arteriogenese.

\section{Schlüsselwörter}

Arteriogenese · AV-Fistel · Schubspannung . Molekulare Mechanismen · Gendefiziente Tiermodelle

\section{Growth stimulation of peripheral and cerebral collaterals to increase intravascular flow in animal models}

\section{Abstract}

Background. Spontaneous collateral growth (arteriogenesis) restores original blood flow only partially because of quick normalization of an initially elevated fluid shear stress. Micro array data from collateral growth in a porcine model with artificially elevated blood flow have increased our knowledge about the molecular mechanisms involved.

Material and Methods. Further molecular analysis requires use of a gene-deficient mouse model. A previously developed method, whereby the fluid shear stress in the peripheral (cerebral) circulation is increased via a cuff anastomosis technique, was transferred to the mouse model. After ligation of the superficial femoral artery (common carotid artery), the peripheral collateral flow was shunted to the femoral vein (jugular vein). To increase cerebral blood flow, we have devel- oped different techniques, including the onesided arteriovenous fistula (solo shunt model) and a two-sided carotid ligature followed by a one-sided arteriovenous fistula (ligature shunt model).

Results. Anastomotic techniques to increase the flow rate and fluid shear stress have been successfully transferred from porcine, rabbit, and rat models to the mouse model. Conclusion. In a gene-deficient mouse model, these new anastomotic techniques allow investigation into the molecular mechanisms involved in collateral growth.

\section{Keywords}

Arteriogenesis - AV fistula · Fluid shear stress . Molecular mechanism · Gene-deficient animal models 


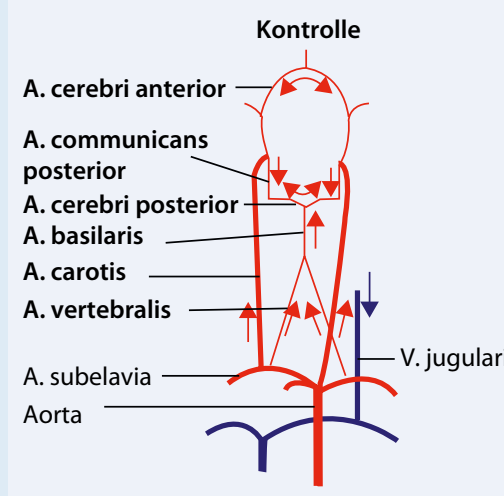

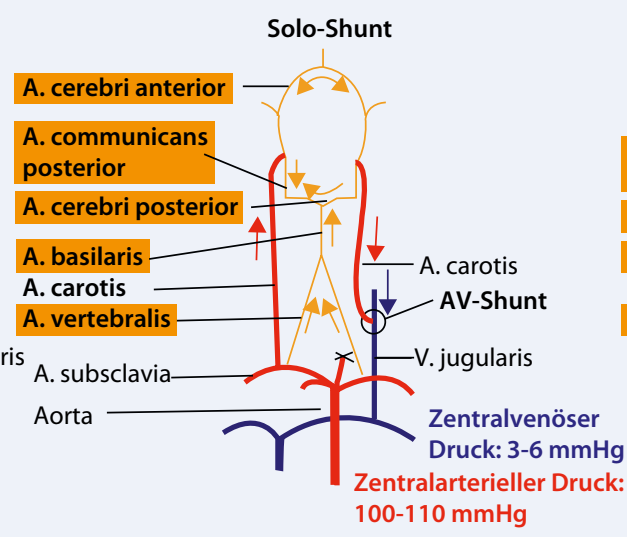

Ligatur-Shunt

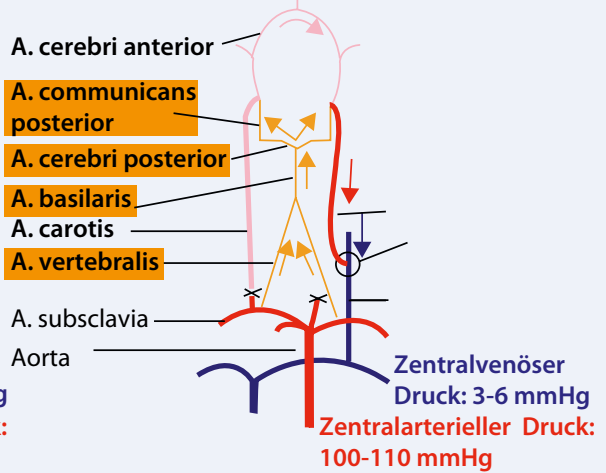

Abb. $1 \Delta$ Schematische Darstellung des physiologischen, zerebralen Blutflusses (Kontrolle) sowie der erwarteten Blutflussänderungen im Solo-Shunt- und Ligatur-Shunt-Modell. Rot: Arterien mit intravasalem Blutfluss; Rosa: ligierte Arterien mit unterbrochenem Blutfluss; Orange: Arterien mit erwarteter Steigerung der Schubspannung

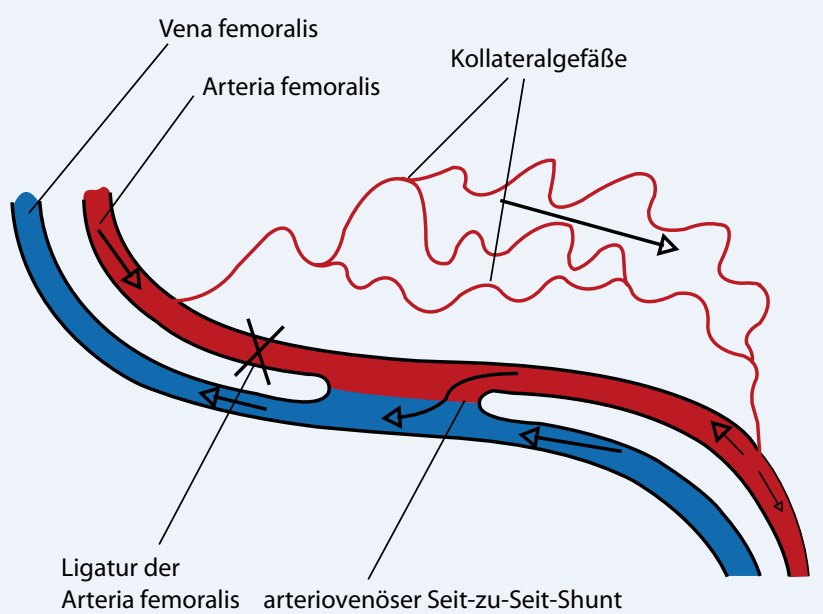

Abb. $2 \Delta$ Schematische Darstellung einer femoralen Nahtanastomose nach proximaler Ligatur der A. femoralis superficialis

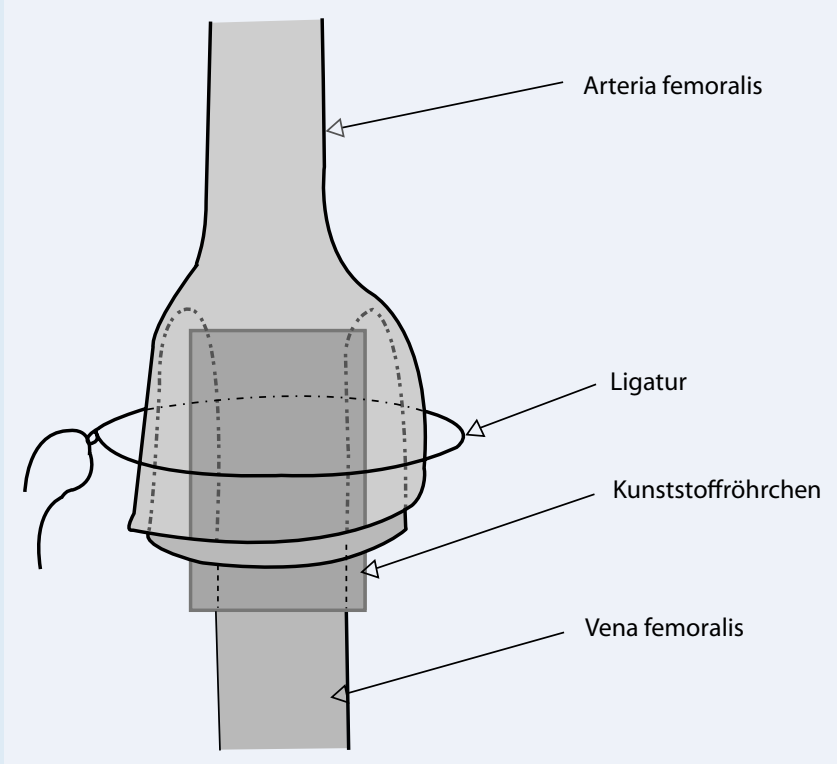

Abb. $3 \Delta$ Schematische Darstellung einer Cuff-Anastomose wendig, unser Modell der chronischen Fluss- und Schubspannungserhöhung auf diese Spezies zu übertragen. Nun stellt die Methode der operativen Shuntanlage zwischen $\mathrm{A}$. und $\mathrm{V}$. femoralis superficialis ein mikrochirurgisch anspruchsvolles Verfahren dar. Je kleiner das Tiermodell, desto höher die Verschlussrate dieser genähten, arteriovenösen Seit-zuSeit-Fisteln. Bei Ratten muss man auch bei mikrochirurgisch erfahrenen Operateuren mit einer Thrombosierungsrate von rund $30 \%$ rechnen, da bereits kleinste technische Fehler wie z. B. Intimaflaps oder ein zu lang andauerndes Klemmen der V. femoralis den vollständigen Verschluss der Fistel innerhalb von Minuten bis Stunden zur Folge haben. Bei Mäusen ist eine Nahtverbindung der Gefäße in der Leiste nahezu unmöglich, auch die Nahtverbindung von A. carotis und V. jugularis ist nicht sicher durchführbar. In den letzten Jahren hat sich ein Verfahren der End-zu-End-Anastomosierung besonders in der Transplantationschirurgie in Tiermodellen durchgesetzt $[7,8,9,12]$. Dieses Verfahren, auch Cuff-Anastomose genannt, verbindet zwei Gefäßstümpfe nahtlos und schafft eine Endothel-aufEndothel-Verbindung.

\section{Material und Methoden}

\section{Tiermodelle}

Alle Studien wurde mit der Genehmigung des Regierungspräsidiums Darmstadt, Hessen nach $₫ 8$ Abs. 1 des deutschen Tierschutzgesetzes durchgeführt. Die Untersuchungen erfolgten in Übereinstimmung mit dem „Guide of Care and Use of Laboratory Animals", der von den „US National Institutes of Health" publiziert wurde (NIH Publication No. 85-23, revised 1996). 
Sämtliche operative Maßnahmen wurden in Peritonealanästhesie durchgeführt. Zusätzlich wurde eine Schmerztherapie mit Buprenorphin s.c. angewandt.

\section{Zerebrales Shuntmodell}

Männliche Sprague-Dawley-Ratten (250300 g, Charles River Laboratories, Sulzfeld) werden in Kontroll-, Solo-Shuntund Ligatur-Shunt-Gruppen operiert. In der Solo-Shunt-Gruppe wird die A. carotis communis auf der linken Seite ligiert, abgesetzt und der distale Stumpf anschließend End-zu-Seit mit der benachbarten Jugularvene anastomosiert und so eine arteriovenöse (AV-)Fistel angelegt. In der Ligatur-Shunt-Gruppe erfolgt zunächst die beidseitige Ligatur der A. carotis communis. 48 Stunden später wird dann auf der linken Seite ebenfalls eine AV-Fistel, wie zuvor beschrieben, angelegt. Bei den Kontrolltieren wird eine Scheinoperation durchgeführt (• Abb.1). Tiere, die in der postoperativen MR-Kontrolle ein zerebrales Ödem oder einen Verschluss der AV-Fistel zeigen, werden von der Studie ausgeschlossen und euthanasiert. Zwischenzeitlich wurde in der Anastomosentechnik die Nahttechnik durch die CuffTechnik ersetzt.

\section{Peripheres Shuntmodell}

Seit-zu-Seit-Anastomose in Nahttechnik (- Abb. 2):

Nach Präparation der A. und V. femoralis werden diese jeweils nach proximal und distal abgeklemmt und jeweils über $5 \mathrm{~mm}$ längs inzidiert. Es erfolgt nun die Naht der arteriovenösen Anastomose in fortlaufender Technik mit einem Polyamid-10:0-Faden zunächst der Hinterwand und nach Fadeneinschlag der Vorderwand. Nach Beendigung der Naht wird zunächst die distale A. femoralis freigegeben; es erfolgen gegebenenfalls Nachstiche. Danach werden alle weiteren Gefäßklemmen entfernt und die Strohmbahn gänzlich freigegeben. Nach einigen Minuten erfolgt die Ligatur der proximalen, zuführenden Arterie, ohne den Abgang der A. femoralis profunda einzuengen. Die Durchgängigkeit der arteriovenösen Fistel wird mittels Dopplerflussmessung überprüft. Auch diese Technik wurde durch die Cuff-Technik ersetzt.

Tab. 1 Veränderungen des Blutflusses im Circulus arteriosus Willisii ( $\mathrm{ml} / \mathrm{min}$ )

\begin{tabular}{|c|c|c|c|c|c|c|}
\hline Modell & \multicolumn{3}{|c|}{ A. cerebri posterior rechts } & \multicolumn{3}{|c|}{ A. cerebri posterior links (Fistelseite) } \\
\hline Tag & 1 & 7 & 14 & 1 & 7 & 14 \\
\hline Kontrolle & $0,48 \pm 0,36$ & $0,48 \pm 0,36$ & $0,48 \pm 0,36$ & $0,48 \pm 0,36$ & $0,48 \pm 0,36$ & $0,48 \pm 0,36$ \\
\hline Solo-Shunt & $0,35 \pm 0,20$ & $0,44 \pm 0,14$ & $0,53 \pm 0,37$ & $0,83 \pm 0,26$ & $1,76 \pm 0,41$ & $2,06 \pm 0,83$ \\
\hline $\begin{array}{l}\text { Ligatur- } \\
\text { Shunt }\end{array}$ & $1,06 \pm 0,20$ & $2,38 \pm 0,38$ & $2,61 \pm 0,70$ & $1,18 \pm 0,10$ & $3,60 \pm 0,59$ & $4,95 \pm 0,45$ \\
\hline Mittelwert $\pm S$ & h; n: je 10 (1 & age); n: je 5 ( & age). & & & \\
\hline
\end{tabular}

\section{Cuff-Technik (• Abb. 3, 4, 5)}

Arteria und V. femoralis werden distal des Leistenbandes präpariert. Ungefähr auf halber Strecke zwischen den dargestellten Abgängen proximal und distal findet sich die Einmündung der V. circumflexa, eines Venenastes, der aus dem präinguinalen Fettgewebe und der Haut in die V. femoralis einmündet und der bei Sprague-Dawley-Ratten selten anatomische Variationen aufweist. Es erfolgt nun die Präparation und die mündungsferne Ligatur dieses venösen Seitenastes. Der Seitenast wird direkt im Mündungsbereich mit einer Mikrogefäßklemme abgeklemmt und unmittelbar proximal der Ligatur abgesetzt, sodass ein langstreckiger Stumpf entsteht. Die A. femoralis wird nach proximal ligiert, ohne den Abgang der A. profunda femoris zu okkludieren. Nun erfolgt das Abklemmen der A. femoralis nach distal und das Absetzten des Gefäßes direkt distal der Ligatur. Auch hier entsteht somit ein langstreckiger Stumpf. Der Venenstumpf wird nun mit einem 10:0-Polyamidfaden angeschlungen und durch ein $1 \mathrm{~mm}$ langes Kunststoffröhrchen (PETubing, Innendurchmesser: $0,4 \mathrm{~mm}$ ) gezogen. Das Kunststoffröhrchen mit dem durchgezogenen Venenstumpf muss nun fixiert werden, um die Vene wiederum um das Röhrchen zu stülpen, sodass nun die Luminalseite evertiert über dem Röhrchen liegt. Im nächsten Schritt stülpt man nun den Arterienstumpf über die evertierte Vene über das Kunststoffröhrchen, es liegt nun Endothel auf Endothel (- Abb.3). Zur Fixierung dieser Endzu-End-Anastomose erfolgt eine Ligatur um die beiden übereinanderliegenden Gefäßstümpfe über dem Kunststoffröhrchen. Das Röhrchen dient als Widerlager dieser Ligatur und liegt streng extraluminal. Die Anastomose der beiden Gefäßstümpfe ist innerhalb des Kunststoffröhrchens durchgängig. Nun werden die beiden Gefäßklemmen geöffnet. Das arterielle Blut des distalen Stumpfes fließt nun über diese End-zu-End-Anastomose in die V. femoralis ab, der distale arterielle Druck ist im Bereich der Fistel auf venöses Niveau reduziert. Die Durchgängigkeit der arteriovenösen Fistel wird mittels Flussmessung mit einer Dopplersonde überprüft.

Dieselbe Technik lässt sich End-zu-End zwischen dem distalen Stumpf der A. carotis interna und der V. jugularis anwenden. Diese Technik ließ sich dann auch auf Mäuse (BALB/c und $\mathrm{C}_{57} \mathrm{BL} / 6$ ) mit einem Gewicht von 25-30 g übertragen.

Der Blutfluss im Circulus arteriosus Willisii (Aa. cerebri posteriores) wurde mehrfach nach Anlage der AV-Fistel mittels quantitativer MR-basierter Flussmessung (MRI-scanner (Bruker PharmaScan 7.0T, $16 \mathrm{~cm}$; Bruker Corporation, Ettlingen - Methode entwickelt von C. Mueller und G. Bachmann, Klinik für diagnostische Radiologie der Kerckhoff-Klinik, Bad Nauheim) bestimmt. Zur Detektion des Circulus arteriosus Willisii wurde zunächst eine dreidimensionale Phasen-KontrastAngiographie-Sequenz des gesamten Gehirns in koronarer Schnittführung durchgeführt. Die Berechnung der Flussquantifizierungssequenz aus den Bildkoordinaten und Messparametern der vorangegangenen Phasen-Kontrast-Angiographie erfolgte mittels eines speziell hierfür entwickelten Programms. Die Auswertung der Flussquantifizierungsdaten erfolgte mit dem ROI-Tool von Paravision 4.1 (Bruker Corporation), das den Blutfluss als Integral in $\mathrm{ml} / \mathrm{min}$ berechnet (- Abb. 6).

\section{Post-mortem-Angiogramm}

Nach adenosininduzierter Vasodilatation wurde das Gefäßsystem der Versuchstiere mittels $37^{\circ} \mathrm{C}$-warmen Kontrastmittels aus Gelatine, Bariumsulfat und Bromphenolblau (gemäß der Fulton-Formel [4]) per- 


\section{Leitthema}

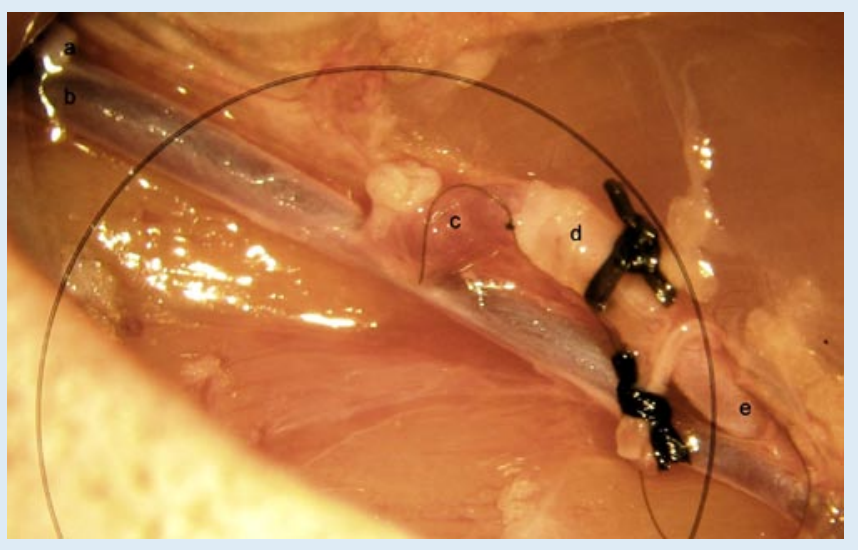

Abb. $4 \Delta$ Bild eines mittels Cuff-Anastomose gebildeten AV-Shunts in der Leiste einer Ratte (a proximal ligierte A. femoralis superficialis; b V. femoralis; c V. circumflexa - anastomosierter Seitenast; $\mathbf{d}$ Cuff-Anastomose; e distale A. femoralis superficialis)

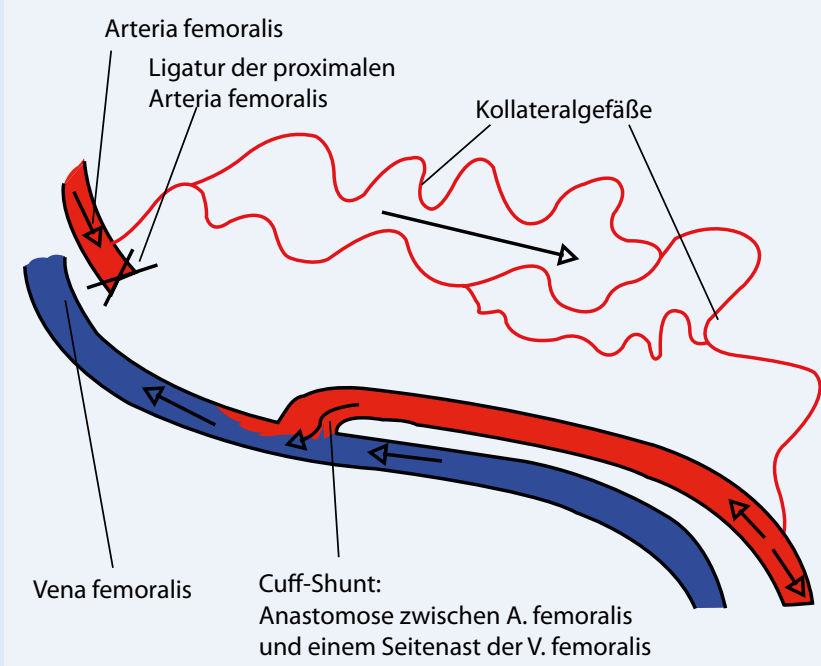

Abb. $5 \Delta$ Schematische Darstellung einer femoralen Cuff-Anastomose nach proximaler Ligatur der A. femoralis superficialis fundiert. Die Injektion des Kontrastmittels erfolgte hierbei bei einem konstanten, physiologischen Druck von $80 \mathrm{mmHg}$. Das Aushärten der Gelatine erfolgte anschließend für 20 min auf Eis. Dann wurde das jeweilige Zielgebiet mit einem CarlZeiss-Mikroskops (OPMI ${ }^{\oplus} 1$ FR) mit Kamera abfotografiert. Durch die adenosininduzierte Vasodilatation sowie die Injektion des Kontrastmittels bei konstantem physiologischem Druck war dabei sicher gestellt, dass auch wirklich die korrekte anatomische Größe der Gefäße abgebildet wurde, sodass dann mittels ImageJ-1.38Software (http://rsb.info.nih.gov/ij/) sowohl die Länge als auch der Durchmesser der Kollateralgefäße als Maß für das Gefäßwachstum bestimmt werden konnten.

\section{Ergebnisse}

\section{Zerebrale Integrität/ Funktionalität der AV-Fistel}

Die zerebrale Integrität wurde am ersten postoperativen Tag mittels MRT kontrolliert. Hierbei zeigten sich keinerlei Auffälligkeiten bei den Tieren der Solo-ShuntGruppe. Auch während der weiteren Beobachtungszeit entwickelte keines der Tiere neurologische Auffälligkeiten. Hingegen fanden sich bei $20 \%$ der Tiere in der Ligatur-Shunt-Gruppe Anzeichen eines zerebralen Ödems, sodass davon auszugehen ist, dass das zerebrale Kollateral- system bei diesem Modell nahe an seine Kapazitätsgrenze gerät.

Die Funktionalität der AV-Fistel wurde durch die quantitative MR-basierte Flussmessung nach 1 Tag, 7 Tagen und 14 Tagen überprüft. Für das Solo-Shunt-Modell betrug der Shuntfluss ca. $3 \mathrm{ml} / \mathrm{min}$, für das Ligatur-Shunt-Modell ca. $1 \mathrm{ml} /$ min. Während des 14-tägigen Beobachtungszeitraums blieb der Shuntfluss weitgehend konstant.

\section{Erhöhung der Flussrate nach AV-Fistel-Anlage}

Nachdem wir die quantitative MR-basierte Flussmessung an das Rattenmodell (Gefäßdurchmesser $<0,5 \mathrm{~mm}$ ) angepasst hatten, wurde 1 Tag, 7 Tage und 14 Tage nach Anlage der AV-Fistel der Blutfluss in den beiden Aa. cerebri posteriores bestimmt (- Tab. 1). In beiden AV-Fistel-Gruppen zeigte sich dabei nach 7 und 14 Tagen im Vergleich zur Kontrollgruppe ein signifikanter Anstieg der intravaskulären Flussrate in den behandelten Aa. cerebri posteriores (• Tab. 1; $\mathrm{p}<0,05)$.

\section{Flussabhängige Stimulierung der Arteriogenese}

Das Wachstum der beiden Aa. cerebri posteriores unter erhöhter Schubspannung in den verschiedenen Modellen wurde sowohl in vivo durch die MRT-
Phasen-Kontrast-Angiographie als auch post mortem durch Kontrastmittelangiogramme nach adenosininduzierter Vasodilatation analysiert, um sicherzustellen, dass durch dieses Verfahren post mortem auch wirklich die In-vivo-Situation abgebildet wird.

Dabei fand sich in beiden AV-FistelGruppen nach 7 und 14 Tagen eine deutliche schubspannungsabhängige Steigerung des Wachstums der Aa. cerebri posteriores sowohl in der Länge als auch insbesondere im Durchmesser (• Abb. 6). Ferner konnte ein signifikanter Unterschied in der Gefäßlänge und dem Gefäßdurchmesser zwischen den verschiedenen Behandlungsoptionen „AV-Fistel - Ligatur - Sham“ nachgewiesen werden (Daten beim Autor).

In der Zusammenschau aller Messdaten zeigte sich eine Korrelation zwischen der Gefäßlänge und dem Blutfluss sowie dem Gefäßdurchmesser und dem Blutfluss mit hoher Übereinstimmung $\left(\mathrm{R}^{2}=0,90 / 0,96-\right.$ Daten beim Autor).

\section{Ergebnisse der Cuff-Technik}

Die technische Modifikation der Anastomosen mittels Cuff-Technik zeigten für die zerebrale Zirkulation und gleichermaßen für die periphere Anwendung im Rattenmodell eine hohe Zuverlässigkeitsrate. Bei insgesamt 10 Anastomosen kam es in keinem Fall zu einer Frühthrombose. Im 

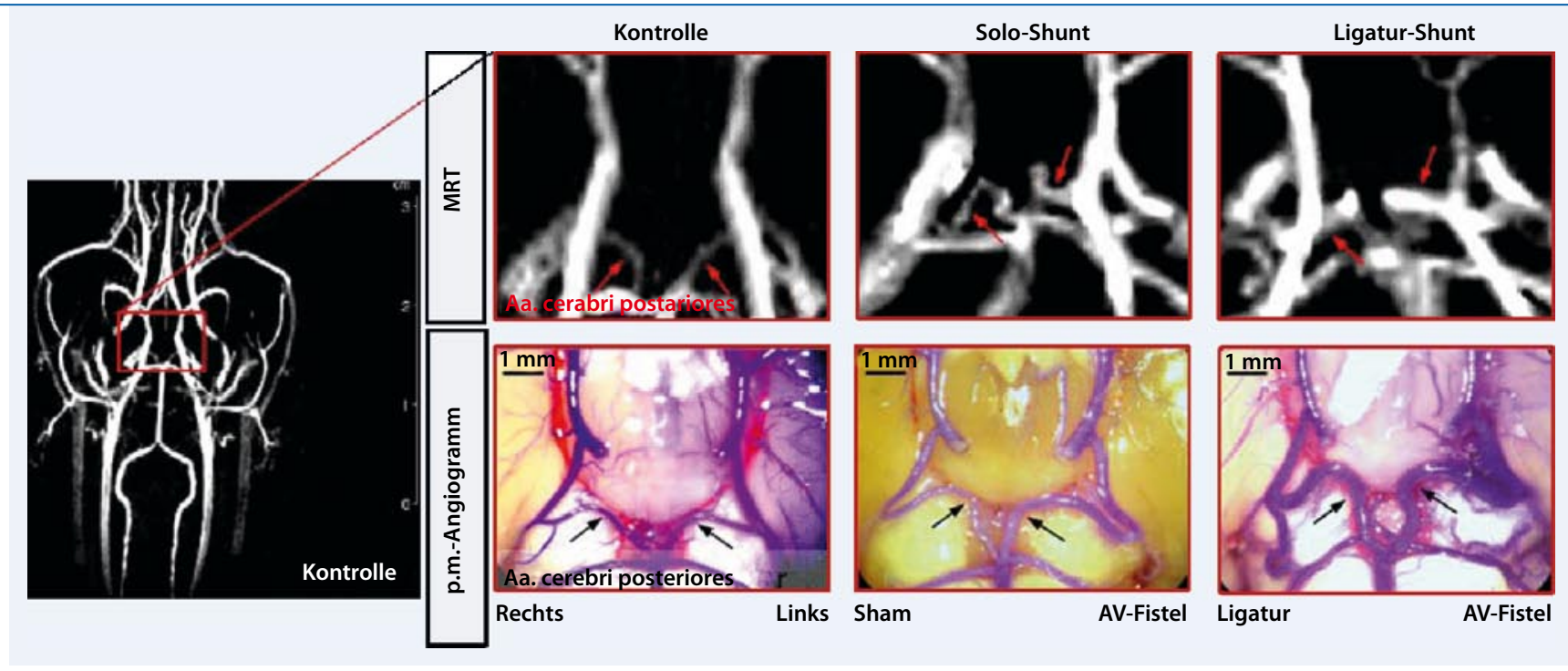

Abb. 6 \& Repräsentative MR-Phasen-Kontrast-Angiographien und Post-mortem-Angiogramme 14 Tage nach Anlage der AV-Fistel in den verschiedenen Behandlungsgruppen zur Evaluierung des Wachstums der zerebralen Kollateralarterien unter erhöhter Schubspannung. (MRT-Bilder mit freundlicher Genehmigung von C. Mueller und G. Bachmann, Klinik für diagnostische Radiologie der KerckhoffKlinik, Bad Nauheim)

Mausmodell ist es ebenfalls mehrfach gelungen, eine Anastomose zwischen dem distalen Stumpf der A. carotis interna und der V. jugularis herzustellen.

\section{Diskussion}

In Analogie zu den Beobachtungen im Bereich des koronaren und peripheren Gefäßsystems, ist die Ausbildung zerebraler Kollateralarterien ein kritischer Faktor zur Reduzierung zerebraler Ischämien sowie des Schlaganfallrisikos im Rahmen zerebrovaskulärer Erkrankungen. Neben den leptomeningealen Anastomosen ist insbesondere der Circulus arteriosus Willisii von zentraler Bedeutung für die zerebrale Integrität bei Patienten mit Stenosen und Verschlüssen im Bereich der supraaortalen Äste.

Da unsere Arbeitsgruppe in vorangegangenen Untersuchungen nachweisen konnte, dass die erhöhte Schubspannung die treibende Kraft der peripheren Arteriogenese ist und zu einem deutlich gesteigertem Kollateralarterienwachstum führt [3], testeten wir kürzlich, ob eine erhöhte Schubspannung auch die treibende Kraft der zerebralen Arteriogenese ist.

Der entscheidende Schritt zur Erhöhung der Schubspannung in unseren bisherigen Modellen lag dabei in der Anlage einer AV-Fistel zwischen dem arteriellen und venösen Gefäßsystem [3]. Auch die Fistelanlage zwischen der A. carotis communis und der $\mathrm{V}$. jugularis ist in der Literatur bereits beschrieben. Allerdings wurden diese AV-Fisteln bisher nur verwendet, um das Remodelling der A. carotis communis bei erhöhter intravasaler Flussrate zu untersuchen. Ferner wurden diese AV-Fisteln zwischen der offenen A. carotis communis und der $\mathrm{V}$. jugularis angelegt und wiesen damit einen viel höheren Shuntfluss auf.

Die AV-Fistel beim Solo-Shunt- und Ligatur-Shunt-Modell wurden dagegen zwischen dem abgesetzten, distalen Ende der A. carotis communis und der Jugularvene angelegt, um die Einspeisung des Flusses über die hintere Hirnzirkulation zu erhöhen. Da wir die quantitative MR-basierte Flussmessung an das Rattenmodell anpassen konnten, war unsere Arbeitsgruppe unseres Wissens nach die erste, die die carotido-juguläre AV-Fistel nutzte, um den Effekt einer erhöhten intravasalen Flussrate auf das Remodelling der intrazerebralen Kollateralarterien zu untersuchen.

Der Begriff „Arteriogenese“ ist definiert als die Entwicklung präexistenter arterioarteriolarer Anastomosen außerhalb ischämischer Regionen zu funktionellen Gefäßen, die die Funktion größerer Gefäße komplett ersetzen können [1,3,
11]. Für das Gehirn trifft diese Definition am ehesten auf die beiden Aa. cerebri posteriores zu - Kollateralarterien, die unter physiologischen Bedingungen nur einen marginalen Blutfluss aufweisen, sich dann aber bei Stenosen und Verschlüssen, insbesondere der A. carotis, stark vergrößern und so die suffiziente Durchblutung des Gehirns weiterhin sicherstellen können.

In unseren Untersuchungen zeigte sich dabei, dass der Durchmesser der A. cerebri posterior und der jeweils gemessene Blutfluss in Korrelation stehen. Auch das Längenwachstum der Aa. cerebri posteriores war vom Blutfluss abhängig, wobei die wachsenden Kollateralgefäße zusätzlich die für Kollateralen typische Korkenzieherformation ausbildeten. Wir konnten somit an der Ratte erfolgreich ein experimentelles Modell zur Erhöhung der intravasalen Flussrate und damit der Schubspannung im Bereich der zerebralen Kollateralzirkulation etablieren und nachweisen, dass das Wachstum zerebraler Kollateralarterien durch eine Erhöhung der intravasalen Flussrate deutlich gesteigert werden kann.

Das in der Cuff-Anastomosentechnik liegende Potenzial hat uns technisch weitergebracht. Wir konnten diese Technik an Sprague-Dawley-Ratten sowohl im Hirn- wie peripheren Modell sicher und zuverlässig durchführen. In allen Fällen 


\section{Hier steht eine Anzeige.}

Springer gelang die Anastomosierung in der oben beschriebenen End-zu-End-Technik und in allen Fällen war die Fistel im Verlauf einer Woche nicht thrombosiert. Unsere bisherigen Beobachtungen zeigten, dass dieses Verfahren mit rund 30 min schneller als die Seit-zu-Seit-Anastomosierung durchführbar war. Der Nutzen kann bei kürzerer Schnitt-Naht-Zeit in einer Reduktion des Narkosestresses auf Seiten der Versuchstiere liegen. Die Cuff-Anastomosentechnik ist für mikrochirurgisch Geübte nach unseren Erfahrungen schnell und einfach zu erlernen. Die Anwendbarkeit der Cuff-Technik an den Halsgefäßen von Mäusen wird uns die Möglichkeit der Nutzung gendefizienter Tiere ermöglichen.

\section{Fazit für die Praxis}

Zusammenfassend konnten wir zeigen, dass das Wachstum der zerebralen Kollateralarterien mit der Zunahme der intravasalen Flussrate korreliert. Unsere Arbeitsgruppe konnte damit erstmalig demonstrieren, dass die Schubspannung nicht nur die treibende Kraft der peripheren, sondern auch der zerebralen Arteriogenese ist. Ferner konnte das zunächst in größeren Tieren etablierte Konzept der chronischen Erhöhung der Schubspannung auf Ratten- und sogar Mausmodelle übertragen werden. In weiteren Studien kann nun der komplette Mechanismus der Steuerung des Kollateralarterienwachstums aufgedeckt werden, um so neue Konzepte zur Therapie arterieller Verschlusskrankheiten zu entwickeln.

\section{Korrespondenzadressen}

\section{Prof. Dr. T. Schmitz-Rixen}

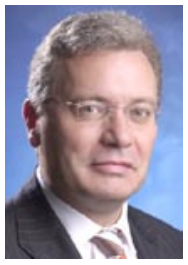

Klinik für Gefäß- und Endovaskular-Chirurgie, GoetheUniversität Frankfurt am Main Theodor-Stern-Kai 7, 60590 Frankfurt am Main schmitz-rixen@em.unifrankfurt.de

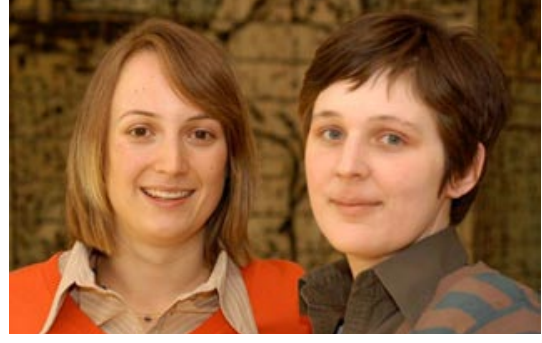

\section{H. Wustreck, W. Schierling}

Arteriogenese - Forschungsgruppe des MPI für Herz- und Lungenforschung, Bad Nauheim

Interessenkonflikt. Der korrespondierende Autor gibt an, dass kein Interessenkonflikt besteht.

\section{Literatur}

1. Carmeliet $P$ (2000) Mechanisms of angiogenesis and arteriogenesis. Nat Med 6:389-395

2. Deindl E, Buschmann I, Hoefer IE et al (2001) Role of ischemia and of hypoxia-inducible genes in arteriogenesis after femoral artery occlusion in the rabbit. Circ Res 89:779-786

3. Eitenmuller I, Volger O, Kluge A et al (2006) The range of adaptation by collateral vessels after femoral artery occlusion. Circ Res 99:656-662

4. Fulton W (1965) The coronary arteries. In: Springfield IL, Charles C (eds) Thomas Publishers

5. Heil M, Clauss M, Suzuki K et al (2000) Vascular endothelial growth factor (VEGF) stimulates monocyte migration through endothelial monolayers via increased integrin expression. Eur J Cell Biol 79:850-857

6. Ito WD, Arras M, Winkler B et al (1997) Monocyte chemotactic protein- 1 increases collateral and peripheral conductance after femoral artery occlusion. Circ Res 80:829-837

7. Matsuura A, Abe T, Yasuura K (1991) Simplified mouse cervical heart transplantation using a cuff technique. Transplantation 51:896-898

8. Nakao A, Tahara K, Inoue S et al (2002) Combined cuff and suture technique for orthotopic whole intestinal transplantation in rats. Microsurgery 22:85-90

9. Reis A, Giaid A, Serrick C, Shennib H (1995) Improved outcome of rat lung transplantation with modification of the nonsuture external cuff technique. J Heart Lung Transplant 14:274-279

10. Sullivan CJ, Doetschman T, Hoying JB (2002) Targeted disruption of the Fgf2 gene does not affect vascular growth in the mouse ischemic hindlimb. J Appl Physiol 93:2009-2017

11. Troidl C, Troidl K, Schierling W et al (2008) Trpv4 induces collateral vessel growth during regeneration of the arterial circulation. J Cell Mol Med [Early View, Date: Nov. 2008]

12. Wu J, Ge X, Fahy GM (2003) Ultrarapid nonsuture mated cuff technique for renal transplantation in rabbits. Microsurgery 23:369-373

13. Yla-Herttuala S, Markkanen JE, Rissanen TT (2004) Gene therapy for ischemic cardiovascular diseases: some lessons learned from the first clinical trials. Trends Cardiovasc Med 14:295-300 